\title{
Valve sparing root replacement: remodeling root repair with aortic ring annuloplasty
}

\author{
Pouya Youssefi ${ }^{1}$, Isabelle Di Centa ${ }^{2}$, Nizar Kheli1 ${ }^{1}$, Mathieu Debauchez ${ }^{1}$, Emmanuel Lansac ${ }^{1}$ \\ ${ }^{1}$ Department of Cardiac Surgery, Institut Mutualiste Montsouris, Paris, France; ${ }^{2}$ Vascular Surgery Unit, Hôpital Foch, Suresnes, France \\ Correspondence to: Pouya Youssefi, BSc(Hons), MBBS, MRCS(Eng), FRCS(CTh), PhD. Department of Cardiac Surgery, Institut Mutualiste \\ Montsouris, Paris, France. Email: pyyoussefi@aol.com.
}

Submitted Feb 18, 2019. Accepted for publication: Apr 08, 2019.

doi: 10.21037/acs.2019.04.01

View this article at: http://dx.doi.org/10.21037/acs.2019.04.01

\section{Introduction}

Recent international guidelines on the management of aortic root disease mention "aortic annuloplasty" in combination with aortic root remodeling as part of a class I indication for management of aortic root aneurysm (1). The remodeling technique provides physiological cusp movement within three reconstructed neo-sinuses, which permits root expansibility through the interleaflet triangles. We have developed a standardized aortic valve and root repair protocol involving a physiological reconstruction of the aortic root using the remodeling technique, cusp effective height resuspension, and a subvalvular external aortic ring annuloplasty (Figure 1).

Here we describe the techniques of valve-sparing root replacement using the remodeling technique and subvalvular annuloplasty for both a tricuspid and bicuspid aortic valve (BAV).

\section{Clinical vignette}

We present two patients who present with NYHA III/IV dyspnoea for the preceding six months. Echocardiography shows the first patient (66 year old gentleman) to have a tricuspid aortic valve (TAV) with grade 4 aortic insufficiency (AI), preserved ejection fraction $(60 \%)$ and a dilated left ventricle with end-diastolic and end-systolic diameters of 75 and $54 \mathrm{~mm}$ respectively. Gated CT reveals sinuses of Valsalva measuring $52 \mathrm{~mm}$ and ascending aorta $48 \mathrm{~mm}$ in diameter. The second patient is a 59-year-old gentleman with a BAV (fusion of the left and right coronary cusps). Echocardiography shows grade $4 \mathrm{AI}$, preserved ejection fraction $(55 \%)$ but dilated left ventricle (end-diastolic diameter $73 \mathrm{~mm}$ and end-systolic diameter $50 \mathrm{~mm}$ ). Gated CT shows sinuses of Valsalva of $55 \mathrm{~mm}$ and ascending aorta of $49 \mathrm{~mm}$ in diameter.

\section{Surgical techniques}

\section{Preparation \& exposition}

The position of the patient is supine on the operating table. A sandbag may be used posterior to the shoulders in order to increase exposure to the aortic root and ascending aorta. Either a median sternotomy or a mini-sternotomy are performed with subsequent heparinisation. Arterial cannulation of the aorta is made high up in the arch in order to maximise the length of excised ascending aorta. Venous cannulation of the right atrium is carried out, with diastolic arrest achieved using direct antegrade Custodial cardioplegia.

\section{Operation}

The ascending aorta is opened and excised down to the STJ. The first step is to dissect around the aortic root down to the subvalvular plane, aiming to reach below the level of the annulus (i.e., below the nadir of the 3 cusps). This can be performed using cautery or scissors. The dissection is usually started at the non-coronary sinus. The non-coronary sinus is dissected away from the roof of the left atrium. This plane is usually well developed and the dissection carried out with ease in order to reach the plane below the nadir of the non-coronary cusp. Subsequent excision of the noncoronary sinus is carried out.

Next, the plane between the left coronary sinus and the 


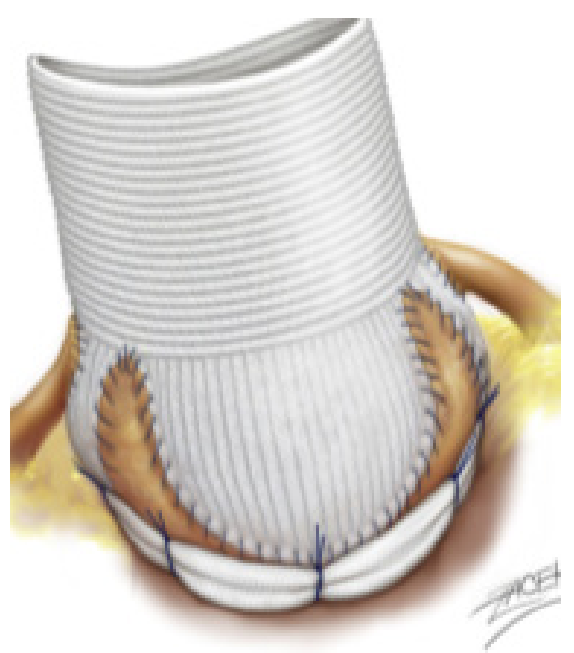

Figure 1 Remodeling root repair with external aortic ring annuloplasty (drawing with kind permission of Dr. Pavel Zacek).

Table 1 Sizing algorithm for the calibrated expansile annuloplasty ring (Extra-Aortic, Coroneo Inc., Montreal, QC, Canada) and remodeling graft (Valsalva graft, Vascutek Ltd, Glasgow, UK) based on Hegar dilator measurement of aortic annulus size

\begin{tabular}{lll}
\hline $\begin{array}{l}\text { Aortic annulus diameter } \\
\text { (Hegar dilator, } \mathrm{mm})\end{array}$ & $\begin{array}{l}\text { Valsalva graft } \\
(\mathrm{mm})\end{array}$ & $\begin{array}{l}\text { Extra aortic ring } \\
(\mathrm{mm})\end{array}$ \\
\hline $25-27$ & 26 & 25 \\
$28-30$ & 28 & 27 \\
$31-35$ & 30 & 29 \\
$\geq 36$ & 32 & 31 \\
\hline
\end{tabular}

pulmonary artery is dissected. In dissecting this plane, it is important to identify the aortopulmonary ligament and to divide it, as doing so will enable the subvalvular plane to be reached. Excision of the left sinus is carried out and the left coronary button is prepared. Further dissection is carried out down to the subvalvular plane. Lastly, excision of the right coronary sinus is carried out, followed by preparation of the right coronary button. One of the more technically difficult sections of the subvalvular dissection step is to dissect the plane between the right sinus and the RV outflow tract/infundibulum. To avoid injuring the infundibulum and entering the $\mathrm{RV}$, dissection must stay parallel and close to the aortic wall. This dissection plane is often more challenging in BAVs. The extent to which dissection can be carried down to the subvalvular level is restrained by the boundary between the right-non commissure and the membranous septum, as it is difficult to dissect to the subvalvular plane in this location. Dissection here is performed with scissors in order to avoid burn injury to the Bundle of His. For all sinuses, a $3 \mathrm{~mm}$ rim of aortic tissue is maintained from the hinge-point of the cusp, as well as $3-5 \mathrm{~mm}$ above each commissure.

A full valve assessment is now carried out. The leaflets are inspected for mobility and quality of tissue, as well as areas of calcification and fenestrations. In the case of BAV, raphes are inspected for thickness and restriction of leaflet mobility.

Geometric height is measured using a ruler. Retraction of cusps is a risk factor for future failure of the repair. A cusp is deemed to be retracted if the geometric height is $<16 \mathrm{~mm}$ in the case of TAV, and $<19 \mathrm{~mm}$ in the case of BAV (in the non-fused cusp). The annulus size is measured using a Hegar dilator. Based on this annulus measurement, the size of the synthetic graft and the expansile external annuloplasty ring (Extra-Aortic, Coroneo Inc., Montreal, QC, Canada) is chosen (see Table 1).

The next step is to place the subvalvular sutures for the annuloplasty ring. Typically, six "U" sutures (Ethicon 3/8 $25 \mathrm{~mm}$ ) are placed circumferentially in the subvalvular plane, one beneath the nadir of each cusp, and one beneath each commissure. The sutures are placed as pledgetted mattress sutures entering inside the aorta $1-2 \mathrm{~mm}$ beneath the nadir of each cusp. The needle should come out of the aorta externally at the lowest limit of the dissection plane, thereby ensuring that the subsequent ring implanted lies at the subvalvular plane. The "U" sutures placed underneath the commissures at the base of the interleaflet triangles should be inserted at the same depth as those at the nadirs of the cusps. Due to the presence of the membranous septum and the nearby Bundle of His, it is important to avoid damage to the conduction system beneath the right-non commissure. As a result, the $6^{\text {th }}$ suture is not placed internally. Instead it is placed on the wall of the left atrium externally at the deepest plane of dissection (nonpledgetted).

\section{TAV_see Video 1}

Alignment of cusp free edge is now established. This ensures correction of any excess free margin length and full symmetry of all cusps before the remodeling process. Each hemi-cusp is compared to its neighbour in a systematic way. $5 / 0$ polypropylene sutures are placed through the nodules of Arantius and used to retract two adjacent hemi-cusps away from their corresponding commissure to assess if there is 
excess length on any cusp. All cusps are compared to their neighbouring cusp in this manner. Central plication of the free margin is carried out if there is excess length.

Remodeling of the aortic root is undertaken using the sinus-shaped Gelweave Valsalva graft (Vascutek Ltd, Glasgow, UK) according to the sizing algorithm of the annulus (Table 1). The Valsalva graft is scalloped into three symmetrical neo-sinuses (placing the commissures at $120^{\circ}$ ). The graft has a circumferential collar at its base, an axially crimped skirt (to mimic the sinuses of Valsalva), and a body for the ascending aorta. The graft is prepared for the remodeling process by excising the collar, and creating scallops by cutting vertically up the skirt to its transition point with the body.

Using 5/0 polypropylene, the remodeling process is started at the nadir of the left coronary sinus. This anastomosis is continued towards the left-non commissure, but is stopped half-way up the sinus. Subsequently, the anastomosis is started again from the commissure down, and the two sutures are tied. The same steps are carried out for all six hemi-sinuses.

Following root reconstruction, cusp repair is performed by first assessing the cusp effective height using a dedicated cusp caliper (Fehling Instruments, Karlstein, Germany). All three cusps are evaluated in turn to assess for prolapse (residual or induced). Plicating 5/0 polypropylene sutures are placed in the middle of the free edge of cusps with effective height $<9 \mathrm{~mm}$, until $9 \mathrm{~mm}$ effective height is achieved.

\section{$B A V$-see Video 2}

Alignment of the cusp free edge is established before the remodeling process. This ensures symmetry of both cusps. The aim in BAV is to use the non-fused cusp as the reference, and to align the free edge length of the fused cusp with the non-fused cusp. A 5/0 polypropylene suture is placed in the nodule of Arantius of both cusps. One commissure is grasped and retracted whilst the sutures in the nodules of Arantius of both cusps are pulled in the opposite direction, thereby showing any excess of length in the free edge of the fused cusp. If this is present, central plicating 5/0 sutures are used to achieve symmetry.

Aortic root remodeling is undertaken using the sinusshaped Gelweave Valsalva graft (Vascutek Ltd, Glasgow, UK) according to the sizing algorithm the annulus (Table 1). The Valsalva graft is scalloped into two symmetrical neosinuses (placing the commissures at $180^{\circ}$ ). The graft is prepared for the remodeling process by excising the collar, and creating scallops by cutting vertically up the skirt to its transition point with the body.

Using $5 / 0$ polypropylene, the remodeling process is started at the nadir of the fused sinus (i.e., at the raphe), with the anastomosis being performed towards the leftnon commissure. This is stopped half-way up the left hemi-sinus. Subsequently, the anastomosis is started again from the left-non commissure down, and the two sutures are tied. The same steps are carried out for all four hemisinuses.

The second step of cusp repair is now performed after placing commissural traction sutures at $180^{\circ}$ in order to mimic a pressurized root. This consists of assessing the effective height using a dedicated cusp caliper to evaluate any residual or induced cusp prolapse (Fehling Instruments, Karlstein, Germany) (2). Plicating sutures are added on the free edge of the culprit leaflet only if the effective height is significantly lower than $9 \mathrm{~mm}$ in order to preserve as much length as possible of the non-fused cusp, for the long term opening of the valve.

\section{Completion}

The final step is placement of the expansile annuloplasty ring at the subvalvular level. The previously positioned subvalvular "U" sutures are each in turn passed around the ring. Once the ring has been parachuted down, the sutures are tied. The position of the coronary buttons in the new graft is assessed, and they are subsequently anastomosed to the Valsalva graft. The distal aortic anastomosis is carried out. A de-airing drill is performed, and the crossclamp is released. Subsequently the patient is weaned from cardiopulmonary bypass and trans-oesophageal echo is used to assess competency of the repair.

For further description of techniques and surgical management of specific valvular lesion sets, please see http://www.aorticvalverepair.net/.

\section{Comments}

The safety of valve-sparing root surgery (VSRR) using the remodeling technique and subvalvular annuloplasty has been demonstrated by the CAVIAAR trial showing similar 30-day mortality compared to a mechanical Bentall procedure, with a trend towards more major adverse events in the Bentall group (OR 2.52, $\mathrm{P}=0.09)$ (3). At 4 years, there were significant benefits seen in patients undergoing valve repair, with freedom from valve-related death and freedom 
from haemorrhagic events being significantly better in the repair group $(99.1 \%$ vs. $94.3 \%$ freedom from valverelated death $(\mathrm{P}<0.001)$, and $89.2 \%$ vs. $78.3 \%$ freedom from haemorrhagic events $(\mathrm{P}=0.02)$ for repair and replacement respectively). Interestingly, freedom from valve-related reoperation was similar in the repair and replacements groups $(\mathrm{P}=0.2)$ A number of important developments in the techniques of aortic valve repair, such as a calibrated expansile annuloplasty ring as well as cusp effective height assessment, have improved repair results and long-term outcomes for patients. Over our series of 482 patients with aortic valve repair, we have shown on a cohort of 177 patients, 7 years freedom from valve-related re-operation, $\mathrm{AI} \geq$ grade 3 , and major adverse valve-related events of $99.1 \%, 100 \%$ and $96.3 \%$ respectively, with similar results for bicuspid and tricuspid valve repair (4).

The advantages of VSRR compared to mechanical Bentall procedure can be demonstrated by evaluating the results of two meta-analyses by Takkenberg et al. $(5,6)$ VSRR showed an improved late mortality of $1.53 \%$ /year (versus 2.02\%/year for Bentall), with better bleeding, thromboembolism, endocarditis, and major adverse valverelated events in the VSRR group. However there was a higher rate of re-operation in VSRR of $1.32 \%$ /year (versus $0.46 \% / y e a r$ for Bentall). Standardization of VSSR using remodeling root repair with expansible aortic annuloplasty might reduce the risk of reoperation and further improve outcomes compared to valve replacement. Analysis of longterm outcomes along with uniform clinical reporting are greatly needed, and will be made possible by the Aortic Valve repair InternATiOnal Registry (AVIATOR) initiative from the Heart Valve (http://heartvalvesociety.org/ AVIATOR/) (7).

\section{Acknowledgments}

P Youssefi was supported by a SCTS Ethicon Scholarship, the Royal College of Surgeons of England Research
Fellowship and Dunhill Medical Trust Research Fellowship.

\section{Footnote}

Conflicts of Interest: $\mathrm{E}$ Lansac has consultant agreements with CORONEO, Inc. (www.coroneo.com), in connection with the development of an aortic ring bearing the trade name "Extra-Aortic." The other authors have no conflicts of interest to declare.

\section{References}

1. Falk V, Baumgartner H, Bax JJ, et al. 2017 ESC/EACTS Guidelines for the management of valvular heart disease. Eur J Cardiothorac Surg 2017;52:616-4.

2. Schäfers HJ, Bierbach B, Aicher D. A new approach to the assessment of aortic cusp geometry. J Thorac Cardiovasc Surg 2006;132:436-8.

3. Lansac E, Bouchot O, Arnaud Crozat E, et al. Standardized approach to valve repair using an expansible aortic ring versus mechanical Bentall: early outcomes of the CAVIAAR multicentric prospective cohort study. J Thorac Cardiovasc Surg 2015;149:S37-45.

4. Lansac E, Di Centa I, Sleilaty G, et al. Remodeling root repair with an external aortic ring annuloplasty. J Thorac Cardiovasc Surg 2017;153:1033-42.

5. Arabkhani B, Mookhoek A, Di Centa I, et al. Reported Outcome After Valve-Sparing Aortic Root Replacement for Aortic Root Aneurysm: A Systematic Review and Meta-Analysis. Ann Thorac Surg 2015;100:1126-31.

6. Mookhoek A, Korteland NM, Arabkhani B, et al. Bentall Procedure: A Systematic Review and Meta-Analysis. Ann Thorac Surg 2016;101:1684-9.

7. de Heer F, Kluin J, Elkhoury G, et al. AVIATOR: An open international registry to evaluate medical and surgical outcomes of aortic valve insufficiency and ascending aorta aneurysm. J Thorac Cardiovasc Surg 2018. [Epub ahead of print].
Cite this article as: Youssefi P, Di Centa I, Khelil N, Debauchez M, Lansac E. Valve sparing root replacement: remodeling root repair with aortic ring annuloplasty. Ann Cardiothorac Surg 2019;8(3):411-414. doi: 10.21037/ acs.2019.04.01 